\title{
Analytical formulation for the radioactive contaminant released from nuclear power plants
}

\author{
B. E. J. Bodmann ${ }^{1}$, U. Rizza ${ }^{2}$, D. Buske ${ }^{3}$, M. T. Vilhena ${ }^{1}$ \\ \& T. Tirabassi ${ }^{4}$ \\ ${ }^{1}$ Federal University of Rio Grande do Sul, Porto Alegre, Brazil \\ ${ }^{2}$ Institute ISAC, National Research Council, Lecce, Italy \\ ${ }^{3}$ Federal University of Pelotas, Pelotas, Brazil \\ ${ }^{4}$ Institute ISAC, National Research Council, Bologna, Italy
}

\begin{abstract}
In this contribution we discuss analytical modelling of radioactive substance dispersion that results from either controlled or accidental release by nuclear power plants. Our model is based on the three-dimensional advection-diffusion equation with mean wind velocity field from observation. All meteorological parameters are determined using the meso-scale Weather Research and Forecasting (WRF) platform. The obtained solution permits us to simulate radioactive substances dispersion in a turbulent regime in the environment around a power plant, which is of interest for nuclear reactor accident scenarios and their related emergency actions. We discuss the closed form method for solving the equation which is then applied to radioactive substance dispersion. Finally, the results of the model are presented.
\end{abstract}

Keywords: advection-diffusion equation, pollutant dispersion, analytical solution, integral transform.

\section{Introduction}

While the renaissance of nuclear power was motivated by the increasing energy demand and the related climate problem, the recent history of nuclear power, more specifically two disastrous accidents, have forced focus on nuclear safety. Although, experience gathered along nuclear reactor developments has sharpened the rules and regulations that lead to the commissioning of latest generation 
nuclear technology, an issue of crucial concern is the environmental monitoring around nuclear power plants. These measures consider principally the dispersion of radioactive material that either may be released in control actions or in accidents, where in the latter knowledge from simulations guide the planning of emergency actions. In this line the following contribution focuses on the question of radioactive material dispersion after discharge from a nuclear power plant.

The atmosphere is considered the principal vehicle by which radioactive materials that are either released from a nuclear power plant in experimental or eventually in accidental events could be dispersed in the environment and result in radiation exposure of plants, animals and last not least humans. Thus, the evaluation of airborne radioactive material transport in the atmosphere is one of the requirements for monitoring and planning safety measures in the environment around the nuclear power plant. In order to analyze the (possible) consequences of radioactive discharge atmospheric dispersion models are of need, which have to be tuned using specific meteorological parameters and conditions in the considered region. Moreover, they shall be subject to the local orography and supply with realistic information on radiological consequences of routine discharges and potential accidental releases of radioactive substances.

The present work provides a model that allows to implement afore mentioned simulations by the use of a hybrid system. In a first step the local meteorological parameters are determined using the next-generation meso-scale numerical weather prediction system "Weather Research and Forecasting" (WRF). The forecasting system contains a three dimensional data assimilation system and is suitable for applications from the meso- down to the micro-scale. The second step plays the role of simulating the dispersion process in a micro-scale, i.e. in the environment within a radius of several tenth kilometers.

\section{Meso-scale simulation for $K$-closure}

The Eulerian approach is widely used in the field of air pollution studies to model the dispersion properties of the Planetary Boundary Layer (PBL). In this context, the diffusion equation that describes the local mean concentrations $\bar{c}=\bar{c}(\mathbf{r}, t)$ at an event point of interest $(\mathbf{r}, t)=(x, y, z, t)$ arising from any contaminant point source, which may be time dependent, can be written as

$$
\partial_{t} \bar{c}+\mathbf{U} \nabla \bar{c}-\nabla^{T} \mathbf{K} \nabla \bar{c}=S .
$$

Here $\mathbf{U}=(\bar{u}, \bar{v}, \bar{w})^{T}$ is the vector field of the mean wind velocity, the diagonal matrix $\mathbf{K}=\operatorname{diag}\left(K_{x}, K_{y}, K_{z}\right)$ contains the eddy diffusivities and $S$ is a source term, to be determined according to the scenario of interest. In eqn. (1) we tacitly related the turbulent fluxes $\overline{\mathbf{U}^{\prime} c^{\prime}}$ to the gradient of the mean concentration by means of eddy diffusivity (K-theory)

$$
\overline{\mathbf{U}^{\prime} c^{\prime}}=-\mathbf{K} \nabla \bar{c} .
$$

Despite the well known limits, the K-closure is largely used in several atmospheric conditions because it describes the diffusive transport in an Eulerian 
framework where almost all measurements are easily cast into an Eulerian form, it produces results that agree with experimental data as well as any other more complex model, and it is not computationally expensive as higher order closures usually are.

The consistency of the K-approach strongly depends on the way the eddy diffusivity is determined on the basis of the turbulence structure of the PBL and on the model ability to reproduce experimental diffusion data. Keeping the Ktheory limitations in mind many efforts have been made to develop turbulent parametrisations for practical applications in air pollution modelling which reveals the essential features of turbulent diffusion, but which as far as possible preserves the simplicity and flexibility of the K-theory formulation. The aim of this step is to elaborate parametrisations for the eddy diffusivity coefficients in the PBL based on the micro-meteorological parameters that were extracted from mesoscale WRF simulations. The WRF model is based on the Taylor's statistical theory and a model for Eulerian spectra $[1,2]$. The main idea of the proposed spectral model relies on considering the turbulent spectra as a superposition of a buoyant produced part (with a convective peak wavelength) and a shear produced part (with a mechanical peak wavelength). By such a model, the plume spreading rate is directly connected with the spectral distribution of eddies in the PBL, that is with the energy containing eddies of the turbulence.

The WRF Simulator is a meso-scale numerical weather prediction system that features multiple dynamical cores and a 3-dimensional variational data assimilation system. The simulator offers multiple physics options that can be combined in various ways. Since this study focusses on the implementation of an interface with a model for the PBL, orography related features of WRF were of importance, more specifically the Land-Surface and PBL physics options were chosen for the present study. In WRF, when a PBL scheme is activated, a specific vertical diffusion is de-activated with the assumption that the PBL scheme will handle this process. The Mellor-Yamada-Janjic PBL scheme derives the eddy diffusivities coefficients and the boundary layer height from the estimations of the Turbulent Kinetic Energy (TKE) through the full range of atmospheric turbulent regimes [3].

Two grids were used for the WRF meso-scale simulation. The outer grid has an extension of the order of half the earth radius so that a significant part of the large scale geological domain of interest is included. The inner grid is centred at the point of interest, i.e. the centre of the power plant where typically the nuclear reactor is located. The simulation may in principal contain a sequence of days or even months. The micro-meteorological data are extracted at the centre point of the inner WRF grid. The spectral model needs these quantities to calculate the eddy diffusivity coefficients.

On the basis of Taylor's theory Taylor proposed that under the hypothesis of homogeneous turbulence, the eddy diffusivities may be expressed as

$$
K_{\alpha}=\frac{d}{d t}\left(\frac{\sigma_{\alpha}^{2}}{2}\right)=\frac{\sigma_{i}^{2} \beta_{i}}{2 \pi} \int_{0}^{\infty} F_{i}^{E}(n) \frac{\sin \left(2 \pi n t \beta_{i}^{-1}\right)}{n} d n
$$


where $\alpha=(x, y, z)$ and $i=u, v, w, F_{i}^{E}(n)$ is the value of the Eulerian spectrum of energy normalized by the Eulerian velocity variance, and $\sigma_{i}^{2}$ corresponds to the Eulerian variance of the turbulent wind field. Following [4], $\beta_{i}=\left(\frac{\pi U^{2}}{16 \sigma_{i}^{2}}\right)^{\frac{1}{2}}$. For large diffusion travel times $(t \rightarrow \infty)$, the filter function in the integral of eqn. (3) selects $F_{i}^{E}(n)$ at the origin of the frequency space, such that the rate of dispersion becomes independent of the travel time from the source and can be expressed as a function of local properties of turbulence,

$$
K_{\alpha}=\frac{\sigma_{i}^{2} \beta_{i} F_{i}^{E}(0)}{4}
$$

where $F_{i}^{E}(0)$ is the value of the normalised Eulerian energy spectrum at $n=0$. In this way the eddy diffusivity is directly associated to the energy-containing eddies which are the principal contribution to turbulent transport. In order to use eqn. (4) we have to find an analytical form for the dimensionless Eulerian spectrum. We assume here that the spectral distribution of turbulent kinetic energy is a superposition of buoyancy and shear components. Such a TKE model may be evaluated as a good approximation for a real PBL, where turbulent production is due to both mechanisms $[5,6]$. In these conditions we may write the Eulerian dimensional spectrum as $S_{i}^{E}(n)=S_{i b}(n)+S_{i s}(n)$, where the subscripts $b$ and $s$ stand for buoyancy and shear, respectively.

An analytical form for the dimensional spectra in convective turbulence has been reported in [2]

$$
S_{i b}(n)=\frac{0.98 c_{i}\left(\frac{n z}{\bar{u}}\right)}{n\left(f_{m i}^{*}\right)^{\frac{5}{3}}\left(1+1.5 \frac{\frac{n z}{\bar{u}}}{f_{m i}^{*}}\right)} \Psi_{\epsilon b}^{\frac{2}{3}}\left(\frac{z}{z_{i}}\right)^{\frac{2}{3}} w_{*}^{2},
$$

while for mechanical turbulence [7]

$$
S_{i s}(n)=\frac{1.5 c_{i}\left(\frac{n z}{\bar{u}}\right)}{n\left(f_{m i}\right)^{\frac{5}{3}}\left(1+1.5 \frac{\frac{n z}{\bar{u}}}{f_{m i}}\right)} \Phi_{\epsilon s}^{\frac{2}{3}} u_{*}^{2}
$$

where $\Psi_{\epsilon b}=\frac{\epsilon_{b} h}{w_{*}^{3}}$ and $\Phi_{\epsilon s}=\frac{\epsilon \kappa z}{u_{*}^{3}}$ are the dimensional dissipation rate functions, $\epsilon_{b}$ and $\epsilon_{s}$ are the convective and mechanical rate of tke dissipation, $f_{m i}^{*}$ is the normalized frequency of the spectral peaks regardless of stratification and $f_{m i}$ is the reduced frequency with the mean wind speed $\bar{u}$ in the mixing layer.

The dimensionless spectrum $F_{i}^{E}(n)$ in eqn. (4) is obtained by normalizing the dimensional spectra with the total variance, $\sigma_{i}^{2}=\int_{0}^{\infty} S_{i}^{E}(n) d n$, that is

$$
F_{i}^{E}(n)=\frac{S_{i}^{E}}{\sigma_{i}^{2}}=\frac{S_{i b}^{E}(n)+S_{i s}^{E}(n)}{\sigma_{i}^{2}} .
$$

The total wind velocity variance is obtained by the sum of mechanical and convective variances $\sigma_{i}^{2}=\int_{0}^{\infty}\left(S_{i b}^{E}(n)+S_{i s}^{E}(n)\right) d n=\sigma_{i b}^{2}+\sigma_{i s}^{2}$. Making use of 
eqns. (3), (5), (6) and eqn. (7) one ends up with $K_{\alpha}=\frac{\beta_{i}}{4}\left(S_{i b}^{E}(0)+S_{i s}^{E}(0)\right)$, that for the $w$-component becomes

$$
K_{z}=\frac{\beta_{i}}{4}\left(\frac{0.98 c_{w}\left(\frac{z}{\bar{u}}\right)}{n\left(f_{m w}^{*}\right)^{\frac{5}{3}}} \Psi_{\epsilon b}^{\frac{2}{3}}\left(\frac{z}{z_{i}}\right)^{\frac{2}{3}} w_{*}^{2}+\frac{1.5 c_{w}\left(\frac{z}{\bar{u}}\right)}{n\left(f_{m w}\right)^{\frac{5}{3}}} \Phi_{\epsilon S}^{\frac{2}{3}} u_{*}^{2}\right) .
$$

\section{On the advection-diffusion approach}

For a time dependent regime considered in the present work, we assume that the associated advection-diffusion equation adequately describes a dispersion process of radioactive material. Approaches to the advection-diffusion problem are not new in the literature, that are either based on numerical schemes, stochastic simulations or (semi-)analytical methods as shown in [8-10]. In these works all solutions are valid for scenarios with strong restrictions with respect to their specific wind and vertical eddy diffusivity profiles. The authors of $[11,12]$ presented a general approach that solves the multidimensional advectiondiffusion equation with variable wind profile and eddy diffusivity coefficient. The main idea here relies on the discretisation of the PBL in a multi-shell domain, assuming in each layer that eddy diffusivity and wind profile take averaged values. Recently, [13] presented a solution that avoid the stepwise approximation of the previous works by the GILTT approach (Generalized Integral Laplace Transform Technique). In this work we improve further the GILTT approach and report a general analytical solution for the multidimensional problem, assuming that eddy diffusivity and wind profiles are arbitrary functions having a continuous dependence on the vertical and longitudinal spatial variables.

Equation (1) is considered valid in the domain $(x, y, z) \in \Gamma$ bounded by $0<x<L_{x}, 0<y<L_{y}$ (with $L_{x}$ and $L_{y}$ sufficiently large), $0<z<h$ (here $h$ is the boundary layer height) and subject to the following boundary and initial conditions,

$$
\left.\mathbf{K} \nabla \bar{c}\right|_{(0,0,0)}=\left.\mathbf{K} \nabla \bar{c}\right|_{\left(L_{x}, L_{y}, h\right)}=\mathbf{0} ; \quad \bar{c}(x, y, z, 0)=0 .
$$

We consider a point source located at an edge of the domain, so that the source position $\mathbf{r}_{S}=\left(0, y_{0}, H_{S}\right)$ is located at the boundary of the domain $\mathbf{r}_{S} \in \delta \Gamma$ and given by $\bar{c}(0, x, y, z)=Q \delta(x) \delta\left(y-y_{0}\right) \delta\left(z-H_{S}\right)$ where $Q$ is the emission rate, $H_{S}$ the height of the source and $\delta$ represents the Cartesian Dirac delta functional.

\section{The closed form solution}

To solve the problem (1) we reduce the dimensionality by one and thus cast the problem into a form already solved in reference [13]. To this end we apply the integral transform technique in the $y$ variable, and expand the pollutant concentration as

$$
\bar{c}(x, y, z, t)=\mathbf{R}^{T}(x, z, t) \mathbf{Y}(y),
$$


where $\mathbf{R}=\left(R_{1}, R_{2}, \ldots\right)^{T}$ and $\mathbf{Y}=\left(Y_{1}, Y_{2}, \ldots\right)^{T}$ is a vector in the space of orthogonal eigenfunctions, given by $Y_{m}(y)=\cos \left(\lambda_{m} y\right)$ with eigenvalues $\lambda_{m}=m \frac{\pi}{L_{y}}$ for $m=0,1,2, \ldots$. For convenience we introduce some shorthand notations, $\nabla_{2}=\left(\partial_{x}, 0, \partial_{y}\right)^{T}$ and $\hat{\partial}_{y}=\left(0, \partial_{y}, 0\right)^{T}$, so that eqn. (1) now reads:

$$
\begin{aligned}
& \left(\partial_{t} \mathbf{R}^{T}\right) \mathbf{Y}+\overline{\mathbf{U}}\left(\nabla_{2} \mathbf{R}^{T} \mathbf{Y}+\mathbf{R}^{T} \hat{\partial}_{y} \mathbf{Y}\right) \\
& \quad=\left(\nabla_{2}^{T} \mathbf{K}+\left(\mathbf{K} \nabla_{2}\right)^{T}\right)\left(\nabla_{2} \mathbf{R}^{T} \mathbf{Y}\right)+\left(\hat{\partial}_{y}^{T} \mathbf{K}+\left(\mathbf{K} \hat{\partial}_{y}\right)^{T}\right)\left(\mathbf{R}^{T} \hat{\partial}_{y} \mathbf{Y}\right) .
\end{aligned}
$$

Upon application of the integral operator $\int_{0}^{L_{y}} d y \mathbf{Y}[\mathbf{F}]=\int_{0}^{L_{y}} \mathbf{F}^{T} \wedge \mathbf{Y} d y$ where $\mathbf{F}$ is an arbitrary function and $\wedge$ signifies the dyadic product operator, and making use of orthogonality renders the matrix equation:

$$
\left(\partial_{t} \mathbf{R}^{T}\right) \mathbf{B}+\overline{\mathbf{U}}\left(\nabla_{2} \mathbf{R}^{T} \mathbf{B}+\mathbf{R}^{T} \mathbf{Z}\right)=\boldsymbol{\Omega}_{1}(\mathbf{R})+\boldsymbol{\Omega}_{2}(\mathbf{R})+\mathbf{R}^{T}\left(\mathbf{T}_{1}+\mathbf{T}_{2}\right)
$$

where appearing integral terms are

$$
\begin{aligned}
\mathbf{B} & =\int_{0}^{L_{y}} d y \mathbf{Y}[\mathbf{Y}]=\int_{0}^{L_{y}} \mathbf{Y}^{T} \wedge \mathbf{Y} d y \\
\mathbf{Z} & =\int_{0}^{L_{y}} d y \mathbf{Y}\left[\hat{\partial}_{y} \mathbf{Y}\right]=\int_{0}^{L_{y}} \hat{\partial}_{y} \mathbf{Y}^{T} \wedge \mathbf{Y} d y \\
\mathbf{\Omega}_{1} & =\int_{0}^{L_{y}} d y \mathbf{Y}\left[\left(\nabla_{2}^{T} \mathbf{K}\right)\left(\nabla_{2} \mathbf{R}^{T} \mathbf{Y}\right)\right]=\int_{0}^{L_{y}}\left(\left(\nabla_{2}^{T} \mathbf{K}\right)\left(\nabla_{2} \mathbf{R}^{T} \mathbf{Y}\right)\right)^{T} \wedge \mathbf{Y} d y \\
\mathbf{\Omega}_{2} & =\int_{0}^{L_{y}} d y \mathbf{Y}\left[\left(\mathbf{K} \nabla_{2}\right)^{T}\left(\nabla_{2} \mathbf{R}^{T} \mathbf{Y}\right)\right]=\int_{0}^{L_{y}}\left(\left(\mathbf{K} \nabla_{2}\right)^{T}\left(\nabla_{2} \mathbf{R}^{T} \mathbf{Y}\right)\right) \wedge \mathbf{Y} d y, \\
\mathbf{T}_{1} & =\int_{0}^{L_{y}} d y \mathbf{Y}\left[\left(\left(\hat{\partial}_{y}^{T} \mathbf{K}\right)\left(\hat{\partial}_{y} \mathbf{Y}\right)\right]=\int_{0}^{L_{y}}\left(\left(\left(\hat{\partial}_{y}^{T} \mathbf{K}\right)\left(\hat{\partial}_{y} \mathbf{Y}\right)\right)^{T} \wedge \mathbf{Y} d y\right.\right. \\
\mathbf{T}_{2} & =\int_{0}^{L_{y}} d y \mathbf{Y}\left[\left(\mathbf{K} \hat{\partial}_{y}\right)^{T}\left(\hat{\partial}_{y} \mathbf{Y}\right)\right]=\int_{0}^{L_{y}}\left(\left(\mathbf{K} \hat{\partial}_{y}\right)^{T}\left(\hat{\partial}_{y} \mathbf{Y}\right)\right)^{T} \wedge \mathbf{Y} d y .
\end{aligned}
$$

Note, that the integrals $\boldsymbol{\Omega}_{i}$ and $\mathbf{T}_{i}$ depend on the specific form of the eddy diffusivity $\mathbf{K}$. The integrals are general, but for practical purposes and for application to a case study we truncate the eigenfunction space and consider $M$ components in $\mathbf{R}$ and $\mathbf{Y}$ only, though continue using the general nomenclature that remains valid.

In order to discuss a specific case we introduce a convention and consider the average wind velocity $\overline{\mathbf{U}}=(\bar{u}, 0,0)^{T}$ aligned with the $x$-axis. Since the variation of the average wind velocity is slow compared to the time intervals for which the meteorological data are extracted from WRF, we superimpose the solution after rotation in the $x-y$-plane in order to transform every instantaneous solution into the same coordinate frame, i.e. the coordinate frame for $t=0$. By comparison of physically meaningful cases, $\left\|\partial_{x} K_{x} \partial_{x}\right\| \ll|\bar{u}|$, which can 
be understood intuitively because eddy diffusion is observable predominantly perpendicular to the mean wind propagation. The principal aspect of interest in pollution dispersion is the vertical concentration profile, that responds strongly to the atmospheric boundary layer stratification, so that the simplified eddy diffusivity $\mathbf{K} \rightarrow \mathbf{K}_{1}=\operatorname{diag}\left(0, K_{y}, K_{z}\right)$ depends in leading order approximation only on the vertical coordinate $\mathbf{K}_{1}=\mathbf{K}_{1}(z)$. For this specific case the integrals $\boldsymbol{\Omega}_{i}$ reduce to

$$
\begin{gathered}
\boldsymbol{\Omega}_{1} \rightarrow\left(\partial_{z} K_{z}\right)\left(\partial_{z} \mathbf{R}^{T}\right) \mathbf{B} ; \quad \boldsymbol{\Omega}_{2} \rightarrow K_{z}\left(\partial_{z}^{2} \mathbf{R}^{T}\right) \mathbf{B} \\
\mathbf{T}_{1} \rightarrow \mathbf{0} ; \quad \mathbf{T}_{2} \rightarrow-K_{y} \mathbf{\Lambda} \mathbf{B}
\end{gathered}
$$

where $\boldsymbol{\Lambda}=\operatorname{diag}\left(\lambda_{1}^{2}, \lambda_{2}^{2}, \ldots\right)$. The simplified equation system to be solved is then,

$$
\partial_{t} \mathbf{R}+\bar{u} \partial_{x} \mathbf{R}=\left(\partial_{z} K_{z}\right) \partial_{z} \mathbf{R}+K_{z} \partial_{z}^{2} \mathbf{R}-K_{y} \mathbf{\Lambda} \mathbf{R}
$$

by virtue of $\mathbf{B}$ being a diagonal matrix.

Once the problem (14) is solved the solution of problem (1) is well determined. Details on the solution can be found in [13].

\section{Application to the Fukushima-Daiichi accident}

In order to illustrate the suitability of the discussed formulation to simulate contaminant dispersion in the PBL, we evaluate simulate radioactive substance dispersion around the Fukushima-Daiichi power plant.

At the 11th of March, 2011 the Fukushima-Daiichi nuclear power plant accident (coordinates in latitude, longitude: $37^{\circ} 25^{\prime} 17^{\prime \prime} \mathrm{N}, 141^{\circ} 1^{\prime} 57^{\prime \prime} \mathrm{E}$ ) caused considerable radiation leakage into the atmosphere and into the sea. The radioactive pollution of the environment and sea was caused principally by the direct release of contaminated water from the power station. To a lesser extent atmospheric release of the radio-nuclide from the atmospheric plume are carried by the winds over the sea during and after the accident sequence. Shorter-lived radioactive elements, such as Iodine-131 were detectable for a few months (halflive of approximately 80 days). Others, such as Ruthenium-106 and Caesium-134 will still persist in the environment for several years (Caesium-137 has a half-life of approximately 30 years).

In the following we show the results for a sequence of four days from the 12th to the 15th of March. Figure 1 shows some meso-scale meteorological information, that was obtained from WRF. The first plot in Figure 1 corresponds to the situation three hours after the beginning of constant release of radioactive material, the second and third plot correspond to 48 hours and 93 hours after time zero.

In the further we show the radioactive substance concentrations close to the surface around the nuclear power plant. Figure 2 shows distributions for 3 hours, 48 hours and 93 hours after the beginning of the substance release with a logarithmic scale. The centre of the nuclear power plant is located in the centre of the plot, the cost line is almost in the north south direction, that is parallel to the $y$-axis in the plot with the ocean to the right side. Shortly after the beginning of the release 

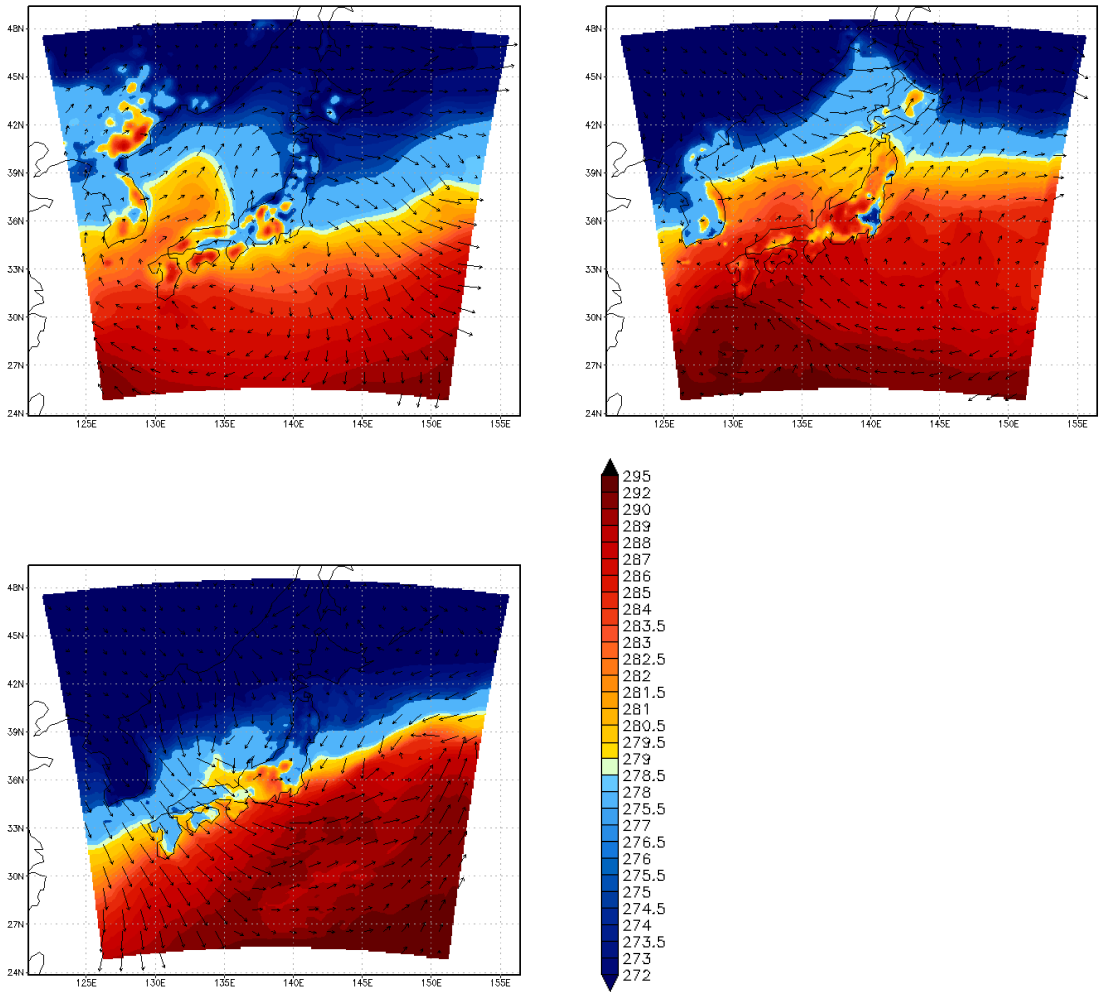

Figure 1: Temperature and mean wind profile from WRF for 3 hours, 48 hours and 93 hours after the beginning of constant release of radioactive substances.

the mean wind pointed towards the ocean, whereas after three days the wind blew towards the south in the direction of Tokyo.

\section{Conclusions}

The present work was based on an Eulerian approach to determine dispersion of radioactive contaminants in the PBL. To this end the diffusion equation was closed by the relation of the turbulent fluxes to the gradient of the mean concentration by means of eddy diffusivity. Since the consistency of the K-approach depends crucially on the determination of the eddy diffusivity considering the turbulence structure of the PBL in its respective stability regimes, we elaborated parametrisations for the eddy diffusivity coefficients based on the micro-meteorological parameters that were extracted from meso-scale WRF simulations, that allowed to take into account the realistic orography of the larger vicinity of a reactor site in consideration. The approach proposed here is based on 

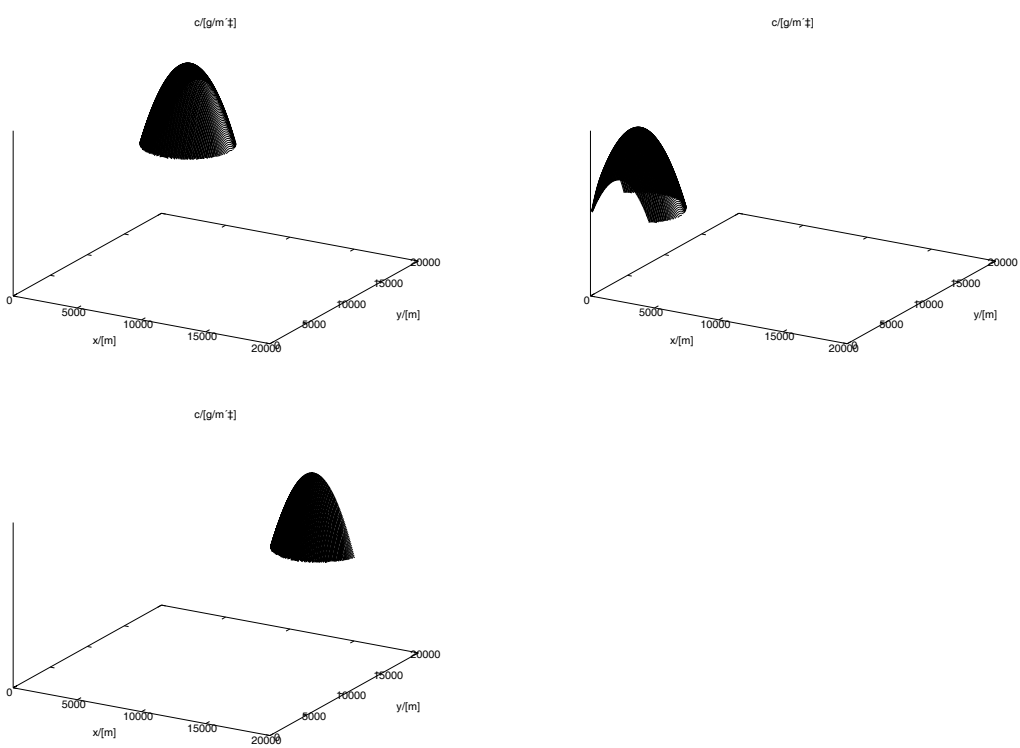

Figure 2: The logarithmic concentration distribution of radioactive substances released from the nuclear power plant for 3 hours, 48 hours and 93 hours after the beginning of the release.

the Taylor statistical diffusion theory and on the spectral properties of turbulence. The assumption of continuous turbulence spectrum and variances, allows the parametrisations to be continuous at all elevations, and in stability conditions ranging from a convective to a neutral condition, and from a neutral to a stable condition so that a simulation of a full diurnal cycle is possible. Simulating micro-meteorology for a short period for the Fukushima Nuclear Power Station Accident may be considered a first step into a direction where the impact of the contamination of radioactive material in the site may be simulated and evaluated for the whole period of the accident until today. Thus the present work may be understood as one tile in a larger program development that simulates radioactive material dispersion using analytical resources, i.e. solutions. In a longer term we intend to build a library that allows to predict radioactive material transport in the PBL that extends from the micro- to the meso-scale.

For model validation one faces the drawback, that the majority of measurements are at ground level, so that one could think that a two dimensional description would suffice, however the present analysis clearly shows the influence of the additional dimension. While in the two dimensional approach the tendency of the predicted concentrations is to overestimate the observed values, this is not the case for the results of the three dimensional description, mainly because it does not assume turbulence to be homogeneous. Moreover the solution of the advection diffusion equation discussed here is more general than shown in the 
present context, so that a wider range of applications is possible. Especially other assumptions for the velocity field and the diffusion matrix are possible.

\section{Acknowledgements}

The authors thank Brazilian CNPq and FAPERGS for the partial financial support of this work.

\section{References}

[1] Olesen, H.R., Larsen, S.E. \& Højstrup, J., Modelling velocity spectra in the lower part of the planetary boundary layer. Bound. Layer Meteor. 29, pp. 285-312, 1984.

[2] Degrazia, G.A., Campos Velho, H.F. \& Carvalho, J.C., Nonlocal exchange coefficients for the convective boundary layer derived from spectral properties. Cont. Atmos. Phys. 70, pp. 57-64, 1997.

[3] Mellor, G.L. \& Yamada, T., Development of a Turbulence Closure Model for Geophysical Fluid Problems. Reviews of Geo. and Space Phys. 20, pp. 851-875, 1982.

[4] Wandel, C.F. \& Kofoed-Hansen, O., On the Eulerian-Lagrangian Transform in the Statistical Theory of Turbulence. J. Geo. Research 67, pp. 3089-3093, 1962.

[5] Højstrup, J., Velocity spectra in the unstable boundary layer. J. Atmos.Sci. 39, pp. 2239-2248, 1982.

[6] Moeng, C.H. \& Sullivan, P.P., A comparison of shear-and buoyancy-driven planetary boundary layer flows. J. Atmos. Sci. 51, pp. 999-1022, 1994.

[7] Degrazia, G.A. \& Moraes, O.L.L., A model for eddy diffusivity in a stable boundary layer. Bound. Layer Meteor. 58, pp. 205-214, 1992.

[8] Demuth, C., A contribution to the analytical steady solution of the diffusion equation for line sources. Atmos. Environ. 12, pp. 1255-1258, 1978.

[9] Nieuwstadt F.T.M. \& de Haan B.J., An analytical solution of onedimensional diffusion equation in a nonstationary boundary layer with an application to inversion rise fumigation. Atmos. Environ. 15, pp. 845-851, 1981.

[10] Tirabassi, T., Operational advanced air pollution modeling. PAGEOPH 160 (1-2), pp. 05-16, 2003.

[11] Moreira, D.M., Vilhena, M.T., Tirabassi, T., Costa, C. \& Bodmann, B., Simulation of pollutant dispersion in atmosphere by the Laplace transform: the ADMM approach. Water, Air and Soil Pollution 177, pp. 411-439, 2006.

[12] Costa, C.P., Vilhena, M.T., Moreira, D.M. \& Tirabassi, T., Semi-analytical solution of the steady three-dimensional advection-diffusion equation in the planetary boundary layer. Atmos. Environ. 40 (29), pp. 5659-5669, 2006.

[13] Moreira, D.M., Vilhena, M.T., Buske, D. \& Tirabassi, T., The state-of-art of the GILTT method to simulate pollutant dispersion in the atmosphere. Atmos. Research 92, pp. 1-17, 2009. 